\title{
Zum dreissigsten Todestag des Neurophysiologen Walter Rudolf Hess
}

\author{
E. Stockhammer
}

\section{Abbildung 1}

Walter Rudolf Hess demonstriert die Atmungsregulation. Quelle: Das Medizinhistorische Museum der Universität Zürich. Zürich: Eigenverlag 1991: 139.

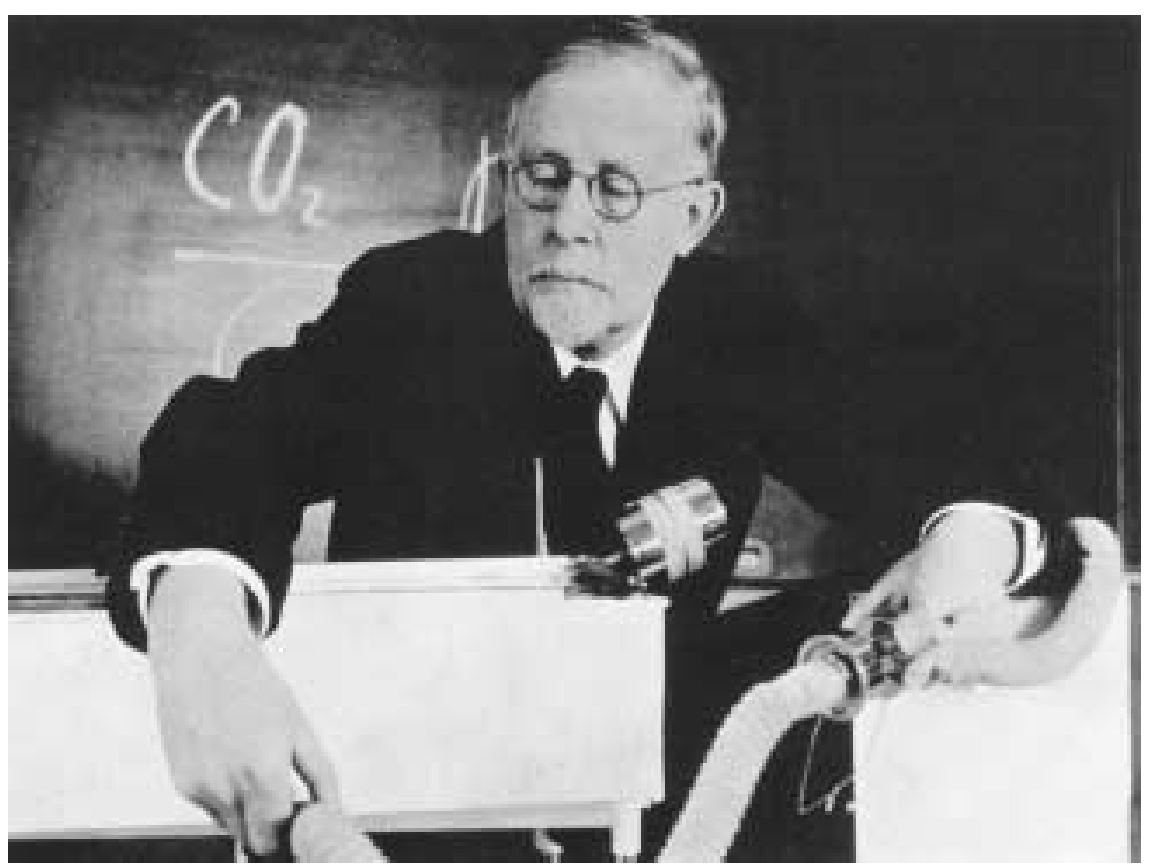

\section{Zusammenfassung}

Vor 30 Jahren verstarb mit dem Nobelpreisträger Walter Rudolf Hess einer der wichtigsten Physiologen und Vertreter der experimentellen Biologie der neueren Zeit. Zu seinen richtungweisenden Hauptverdiensten zählen aus heutiger Perspektive die Einführung einer funktionellen Hirnforschung, die Kartographie des Zwischenhirns, die Aufschlüsselung der Funktionsorganisation des vegetativen Nervensystems und die Beurteilung des Schlafzustandes als aktiven positiven Vorgang, womit Hess - trotz jahrelanger Rückschläge - einen Paradigmenwechsel in der Schlafforschung herbeiführte. Sein Werk hinterliess Spuren in der Grundlagenforschung der gesamten Physiologie und befruchtete darüber hinaus die klinischen Fächer Ophthalmologie, Innere Medizin und Neurologie. Leben und Werk werden kurz umrissen und sollen die anhaltende Bedeutung dieses unkonventionellen «Selfmademan» darlegen.
Das umfangreiche und vielseitige Werk von Walter Rudolf Hess (über 250 Artikel [1] und mehrere Monographien [2-6]) beeindruckt bis heute. Seine Gedankenfolge widerspiegelt sich in der Chronologie der wissenschaftlichen Thematik, die vom peripheren, geschlossenen Organsystem zur funktionellen, zentral gesteuerten Ordnung des Organismus vorrückte. Von der Blutviskosität über die Kreislaufregulierung, die Regulierung der Atmung [2] und der Verdauung führte der Weg zur Erforschung der funktionellen Organisation des vegetativen Nervensystems [3], des Schlafzustandes, der subkortikal programmierten Motorikäusserung bis zur ausführlichen Kartographie des Zwischenhirns als übergeordnete Zentralstelle und Koordinator der inneren Organe [4], wofür Hess 1949 den Nobelpreis erhielt. Im Alter - ähnlich dem grossen Hirnanatomen der Jahrhundertwende Constantin von Monakow (1853 - 1930) - folgte schliesslich die eingehende Beschäftigung mit der «Psychologie in biologischer Sicht» [6], wobei es ihm, im Gegensatz zu Monakow, gelingt, Naturwissenschaft und Moralphilosophie nicht zu vermischen $[7,8]$.

\section{Wissenschaftliche Karriere [9]}

1881 kam Walter Rudolf Hess in Frauenfeld zur Welt und wuchs als Sohn einer Arztgehilfin und eines Physiklehrers in einem Elternhaus auf, das sowohl sein Interesse an der Naturwissenschaft förderte als auch seine später so hilfreiche technische Begabung unterstützte. Nach dem Medizinstudium an den Universitäten Lausanne, Bern, Berlin, Kiel und Zürich entschied sich Hess für eine ophthalmologische Ausbildung bei Otto Haab (1850-1931) in Zürich. Bereits damals interessierte er sich für die Physiologie: In seiner Dissertation befasste er sich mit der Viskosität des Blutes und entwickelte ein neues Untersuchungsinstrument, das Viskosimeter, das jahrelang klinisch in Gebrauch stand. Drei Jahre nach Eröffnung der eigenen Augenpraxis sah sich Hess finanziell in der Lage, seinen innersten Neigungen und Forschungsdrängen nachzugehen. Er liess sich als Assistent im Physiologischen Insti- 
tut Zürich bei Justus Gaule (1849-1939) anstellen und verfasste eine Habilitation über die physikalisch-biologische Basis der Kreislaufregulierung.

Nach anschliessenden Studienaufenthalten 1916 in Bonn beim Physiologen Max Verworn (1863 - 1923) und nach dem Krieg in England bei John Newport Langley (1852-1933), der sein Interesse für das autonome Nervensystem förderte, kehrte Hess in die Schweiz zurück. Bereits 1917 wurde er vom Zürcher Regierungsrat - und wie bei Monakow nicht ohne den Widerspruch der medizinischen Fakultät - als Nachfolger Gaules zum ordentlichen Professor für Physiologie ernannt.

Als Leiter des physiologischen Instituts konnte Hess die eigene Denk- und Arbeitsweise vollumfänglich umsetzen und sie auch in die Lehre einfliessen lassen (Abb. 1). Er vertiefte sich in Forschungsarbeiten über die Regulation von Kreislauf, Atmung und Verdauung, verfolgte deren übergeordnetes Koordinationssystem und beschäftigte sich schliesslich mit dem vegetativen Nervensystem. In einer packenden Monographie weitete Hess die rein anatomische Beschreibung von Sympathikus und Parasympathikus auf «die funktionelle Organisation des vegetativen Nervensystems» [3] aus. Hess unterschied prinzipiell zwei verschiedene funktionelle Grundeinstellungen des vegetativen Nervensystems: die «ergotrope» Einstellung, mit der sich der Organismus gegen die Umwelt behauptet, und die «trophotrope», in der sich der Orga-

Abbildung 2

Affektive Abwehrreaktion einer Katze mit Fauchen, erweiterten Pupillen, zurückgelegten Ohren und gesträubten Rückenhaaren nach hypothalamischer Reizung. Quelle: [5]: 27.

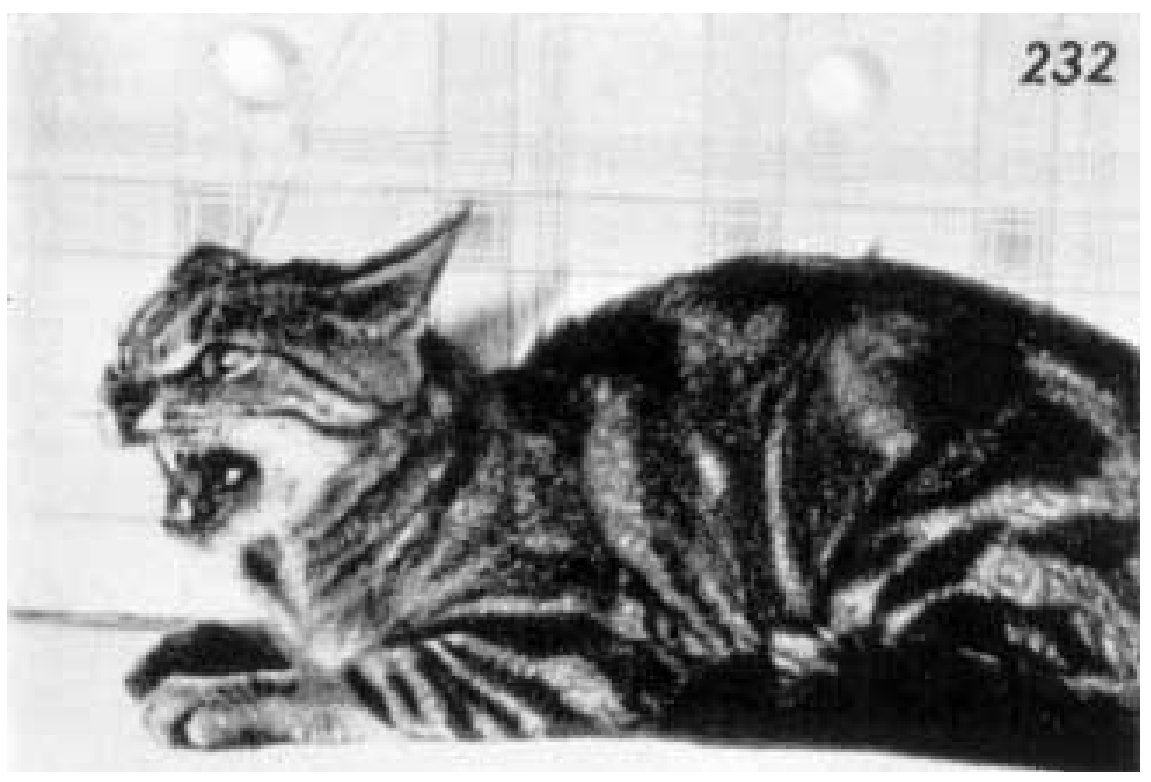

nismus erholt und regeneriert. Sympathikus und Parasympathikus bilden dabei die anatomische Basis dieser beiden antagonistischen Kräfte im dynamischen Gleichgewichtsprozess.

\section{Exploration des Zwischenhirns}

Nach der Entdeckung vieler Kortexfunktionen, etwa der Sprachzentren, in einer Zeit also, in der bei den tonangebenden Hirnforschern eine «kortikozentrische» Konzeption des Zentralnervensystems vorherrschte und die experimentelle Physiologie die In-vivo-Experimente zu Gunsten der In-vitro-Studien zu verlassen begann, beschritt Hess entgegengesetzte Wege: Er wandte sich mit neuen, tierexperimentellen Methoden dem schwer zugänglichen Subkortex zu. Mittels feinster Elektroden reizte Hess am wachen, nicht narkotisierten Tier punktförmig eine eng begrenzte Region, um damit eine Verhaltensreaktion auszulösen, die anschliessend zu Studienzwecken filmisch festgehalten wurde. Systematisch tastete er das Zwischenhirn von oben nach unten und von hinten nach vorn elektrisch ab. Durch die anschliessenden Eintragungen der Reizpunkte auf den Gehirnschnitten entstanden erste detaillierte Karten der Zwischenhirnfunktionen. Auf diesen Funktionskarten finden sich anatomisch klar begrenzte kleinste Hirnareale, durch deren Reizung ein definierter Vorgang wie beispielsweise die Veränderung des Blutdruckes, Auslösung der Defäkation, Induktion des Schlafes oder auch eine koordinierte Reaktion wie das aggressive Verhalten einer Katze mit Pupillendilatation, Pulsanstieg und Aufstellen der Fellhaare provoziert werden kann (Abb. 2). Mit der für diese Pionierarbeit verliehenen Auszeichnung des Nobelpreises erreichte Hess 1949 den Höhepunkt seiner wissenschaftlichen Karriere und wurde international anerkannt.

\section{Die Entdeckung des Schlafzentrums}

In den ausgehenden 1920er Jahren beobachtete Hess, der selbst unter Schlaflosigkeit litt, als Folge von Reizexperimenten im Zwischenhirn wiederholt das Einsetzen eines schlafartigen Zustandes bei seinen Versuchstieren. Er war sich der Bedeutung dieser eher zufällig gemachten Entdeckung wohl bewusst. In den nachfolgenden zwanzig Jahren analysierte er über dreihundert Fälle, die - je nach Stimulationsort - zu verschiedenen schlafähnlichen Zustandsbildern führten. Die eindeutigsten Reaktionen von physiologischem Schlaf, die er von anderen 
Abbildung 3

Sagittalschnitt durch das Zwischenhirn. Die schräge Schraffur gibt das Gebiet des sogenannten Schlafzentrums an. Quelle: [14]: 325.

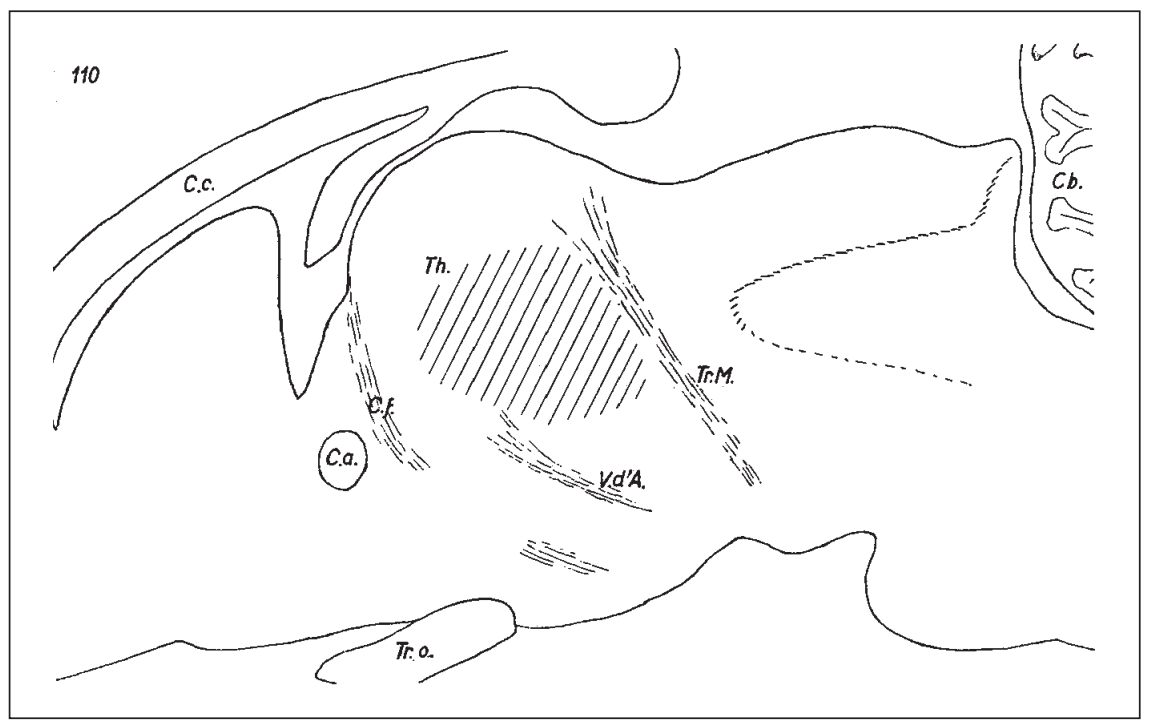

schlafartigen Zuständen unterschied, löste Hess durch Reizungen im Thalamusgebiet aus (Abb. 3) [4, 10 - 12]. Bei hypothalamischen Herden hingegen bemerkte er tagelang anhaltende Somnolenz, ein Befund, der mit den damals vieldiskutierten klinischen Beobachtungen des österreichischen Hirnanatomen Constantin von Economo (1876-1931) und dessen hirnhistologischen Untersuchungen bei Encephalitis lethargica übereinstimmte.

Provokativ für die damalige Zeit und richtungweisend für die Schlafforschung aus heutiger Perspektive war seine Interpretation der Forschungsergebnisse: Entgegen der damals vorherrschenden Auffassung, der Schlaf sei ein Funktionsdefizit, beschrieb Hess den Schlafvorgang als aktiven Zustand bzw. ergotrophe Einstellung. Er fügte den Schlaf-Wach-Mechanismus in die funktionelle Gesamtorganisation des vegetativen Nervensystems ein und ordnete den Schlafzustand der selektiven Aktivität des Parasympathikus zu. Die Existenz des REM-Schlafes, in dem es $\mathrm{zu}$ tonischen und phasischen Veränderungen von Parasympathikus und Sympathikus kommt, war in der damaligen Zeit noch nicht bekannt. Eindrücklich demonstrierte der gelernte Ophthalmologe am Beispiel der Veränderung des Auges im Schlaf (starke Pupillenverengung und aktiver Lidschluss), dass die Aktivität des Sympathikus im Schlaf nicht erlischt, sondern vom Parasympathikus überlagert wird. Diese Augenreaktion wandte Hess unter andern Kriterien als ein physiologisches Kennzeichen des Schlafzustandes an [12].
Hess selbst machte sich das EEG, das ideale Schlafmessgerät schlechthin, in seiner gesamten Forscherzeit nie zunutze. Dabei publizierte der Psychophysiologe Hans Berger (1873-1941) in Jena - ein grosser Anhänger Monakows - bereits 1929 die für die spätere Neurophysiologie und Schlafforschung entscheidende Entwicklung der Elektroenzephalographie [13]. Während Hess Weltruhm erfuhr, wurde Bergers wegweisende Methode zu seinen Lebzeiten kaum zur Kenntnis genommen. Diese Situation, sein eher introvertierter Charakter mit Neigung zur Depression und nicht zuletzt die politische Situation im nationalsozialistischen Deutschland waren wohl mit dafür verantwortlich, dass sich Berger 1938 aus der Berufstätigkeit zurückzog und 1941 den Freitod wählte [14]. Walter Rudolf Hess, dessen Arbeiten von Berger sehr wohl erwähnt wurden, führte das EEG erst in seiner letzten Monographie an [6]. Anfang der 1950er Jahre allerdings wiederholte die Schweizer Forschergruppe Konrad Akert, Werner P. Koella und Rudolf M. Hess, Sohn von Walter Rudolf Hess, die Hessschen Stimulationsversuche unter gleichzeitiger Aufzeichnung der kortikalen und subkortikalen Aktivität mit dem EEG und wiesen die physiologische Antwort als tatsächlichen Schlafzustand nach [15].

\section{Philosophische Aspekte}

Walter Rudolf Hess interessierte sich auch für philosophische Fragen. Vielleicht verbirgt sich dahinter eine Teilantwort auf die unglaubliche Energie und Eigendisziplin, die Hess auszeichneten und ihn unter anderem zum Erfolg führten: Hess scheint bereits in jungen Jahren von der Idee beseelt gewesen zu sein, dass ein Organismus nur Dank einer minuziösen Ordnung hierarchischer Prägung funktionieren könne, wozu das ökonomische Prinzip, mit geringstem Aufwand einen grösstmöglichen Erfolg zu erzielen, die Grundlage bildete. Die Erfassung dieser funktionellen Ordnung in seiner Gesamtheit, ausgehend vom peripheren, teils autonomen Sektor bis hin zur zentralen Koordinationsstelle, wirkte wie eine Triebfeder für all seine Arbeiten. Wie viele andere Hirnforscher der damaligen Zeit vertrat Hess überdies die Meinung, dass «der Aufgabenkreis der Physiologen sich nicht auf die Funktionsorganisation des Einzelindividuums beschränkt», sondern auch Probleme wie «die Organisation der menschlichen Gesellschaft» betreffen solle. In einem 1944 publizierten Artikel «Über die kollektive Ordnung in biologischem Aspekt» [16] überträgt Hess seine physio- 
logischen Erkenntnisse auf die menschliche Gesellschaft. Dabei nimmt er - analog zur funktionellen Ordnung des Einzelorganismus - eine zentralistische Ordnung des Kollektivismus als sozial effizientestes Prinzip an.

Mit dieser an den Biologismus deutscher Naturphilosophie anlehnenden Auffassung steht Hess in einer langen Tradition vieler, insbesondere deutschsprachiger Hirnforscher des 19. und beginnenden 20. Jahrhunderts, die neben ihrer nüchternen Hirnforschung versuchten, das neurologische Modell politisch aufzuladen und als Metapher anzuwenden [8].

\section{Literatur}

1 Hess WR. Gesammelte Arbeiten von Walter Rudolf Hess 1903-1970 in 8 Bänden. Zürich: Medizinhistorisches Institut der Universität. Signatur 12 H 586 AR.

2 Hess WR. Das Zwischenhirn und die Regulation von Kreislauf und Atmung. Leipzig: Thieme; 1932.

3 Hess WR. Die funktionelle Organisation des vegetativen Nervensystems. Basel: Schwabe; 1948.

4 Hess WR. Das Zwischenhirn. Syndrome, Lokalisationen, Funktionen. Basel: Schwabe; 1949 und 2. erweiterte Auflage: 1954.

5 Hess WR. Hypothalamus und Thalamus. Experimental-Dokumente. Stuttgart: Thieme; 1956.
6 Hess WR. Psychologie in biologischer Sicht. Stuttgart: Thieme; 1968.

7 von Monakow C. Gehirn und Gewissen. Psychobiologische Aufsätze. Herausgegeben von W. Corti. Zürich: Conzett \& Huber; 1950.

8 Hagner M (Hrsg.). Ecce Cortex. Beiträge zur Geschichte des modernen Gehirns. Göttingen: Wallstein; 1999.

9 Waser PG. Walter Rudolf Hess. Aus seinem Leben und seiner Tätigkeit an der Medizinischen Fakultät der Universität Zürich. Gesnerus 1981;2:279-93.

10 Hess WR. Das Schlafzentrum. Verh Schweiz Naturf Ges Basel 1927;889:247-8.

11 Hess WR. Der Schlaf. Schweiz Med Wochenschr 1933;112:1-18.

12 Hess WR. Das Schlafsyndrom als Folge dienzephaler Reizung. Helv Physiol Acta 1944;2:305-44.

13 Berger H. Das Elektrenkephalogramm des Menschen. Nova Acta Leop 1938;6:173-309.

14 Gloor P. Hans Berger. Psychophysiology and the discovery of the human electroencephalogram. In: Harris P, Mawdsley C (eds.). Epilepsy: proceedings of the Hans Berger centenary symposium. Edinburgh: Churchill Livingstone; 1974:353-373.

15 Akert K, Koella W, Hess RM. Sleep produced by electrical stimulation of the thalamus. Am J Physiol 1952;168(1):260-7.

16 Hess WR. Über die kollektive Ordnung in biologischem Aspekt. In: Vom Krieg und vom Frieden. Festschrift der Universität Zürich zum siebzigsten Geburtstag von Max Huber. Zürich: Schulthess; 1944:151-72. 